\title{
Electrochemical discharge machining: Principle and possibilities
}

\author{
AMITABHA GHOSH \\ Department of Mechanical Engineering, Indian Institute of Technology, \\ Kanpur 208 016, India \\ Present address: Indian Institute of Technology, Kharagpur 721 302, India \\ e-mail: amitabha@iitkgp.ernet.in
}

\begin{abstract}
This paper highlights the important results of the investigations on Electrochemical Discharge Machining (ECDM) conducted by the author and his coresearchers. It has been found that "switching phenomenon" plays a crucial role in spark generation and not the straightforward breakdown of the non-conducting vapour blanket. The mechanism of spark generation has been understood reasonably well and inductance in the circuit has emerged as an important process parameter from the investigations. This information has been effectively used to improve the process capability of ECDM by a substantial amount. It has also been shown how ECD can be very conveniently used for micro-welding operation without using any sophisticated arrangement.
\end{abstract}

Keywords. Electrochemical discharge machining; switching phenomenon; spark generation; inductance; micro-welding.

\section{Introduction}

Manufacturing is now recognized by all sections of our society as one of the most important activities since it affects very significantly the GNP, international trade and employment. Manufacturing is broadly defined as

"an activity by which material, labour (including information), energy and machines are brought together to produce a product whose value is more than the total value of the individual inputs".

This is schematically indicated in figure 1 . However, this definition is too generalised and one can be more specific stating that

"the activity of manufacturing brings together material, labour, machine and energy to shape and assemble finished products without involving any physical and chemical changes in the materials involved." 


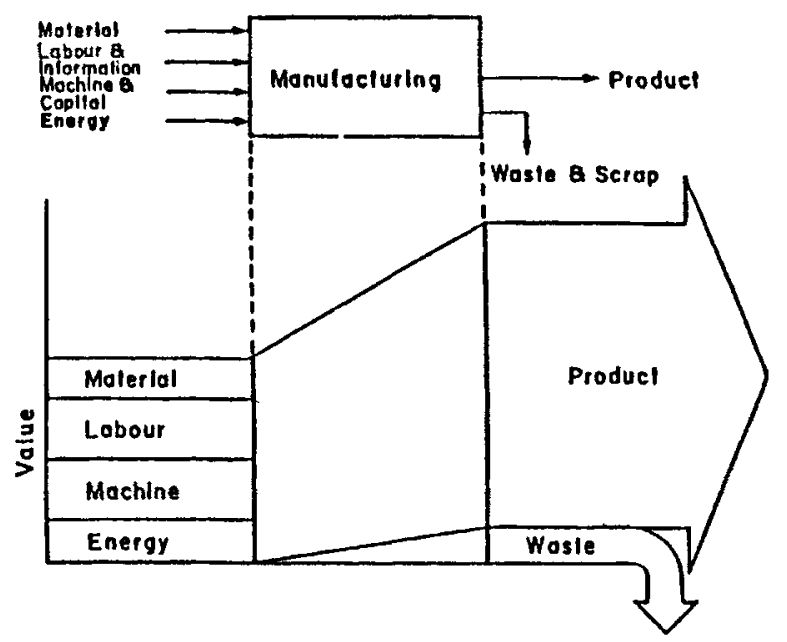

Figure 1. Value addition in manufacturing activity.

"Engineering industry manufacturing" is what we mean by "manufacturing" in the field of Mechanical Engineering. Figure 2 suggests that almost one third of the production in India comes from such activity and, therefore, "mechanical manufacturing" represents a very vital pillar of our economy.

"Machining" has a special status among all processes covering the whole spectrum of "mechanical manufacturing" because of its capabilities as below.

- High accuracy and finish can be achieved.

- Complex shapes can be produced.

- Machining force and power are independent of the work size and shape as all machining processes are basically generating processes.

- The property of the bulk of the work material remains unaffected.

Machining has also been one of the earliest manufacturing processes and is continuously evolving to cope with the new challenges faced by the manufacturing industries.

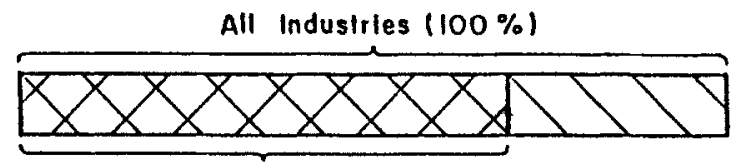

All Manufacturing Induslrles $(77 \%)$

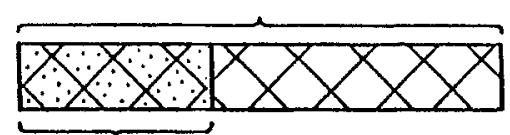

All Englneering

Industries $(31 \%)$

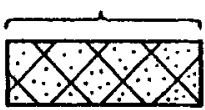

Figure 2. Proportion of engineering industries. 


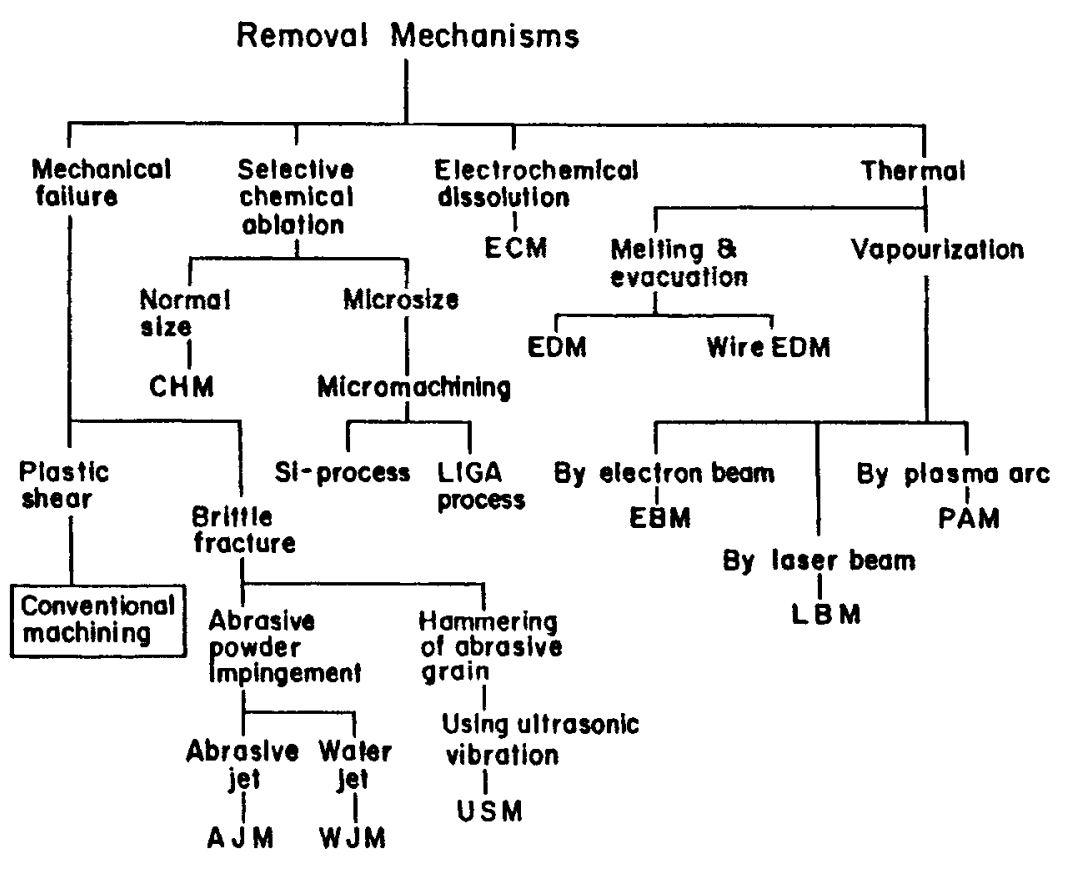

Figure 3. Family of machining processes.

In machining, the required shape, size and finish are obtained by removing excess material in a controlled manner in the form of small chips. In conventional machining removal is in the form of small chips and in many advanced processes chip size is extremely small. Removal of excess material can be achieved in various ways using different forms of energy as shown by the tree diagram in figure 3 .

The two most extensively used unconventional machining processes are

- Electrochemical machining (ECM), and

- Electric discharge machining (EDM).

Considering the different aspects of their capabilities, these two processes are considered to possess maximum potential. However, inspite of their many virtues both these processes suffer from a very important limitation. To employ either ECM or EDM the work material must be electrically conducting. The development of electrochemical discharge machining has taken place in recent years primarily to eliminate this difficulty.

\section{Electrochemical discharge machining}

A study of all machining processes indicates that any scheme for removing material in the form of small particles in a controlled fashion can be used for the shaping of objects. To circumvent material and shape problems, quite often the approach adopted for machining is to cause the melting of a small portion of the workpiece by means of intense localized heat generation. By controlling the location of the heat source in a proper way, the required shape of the workpiece can be achieved. 


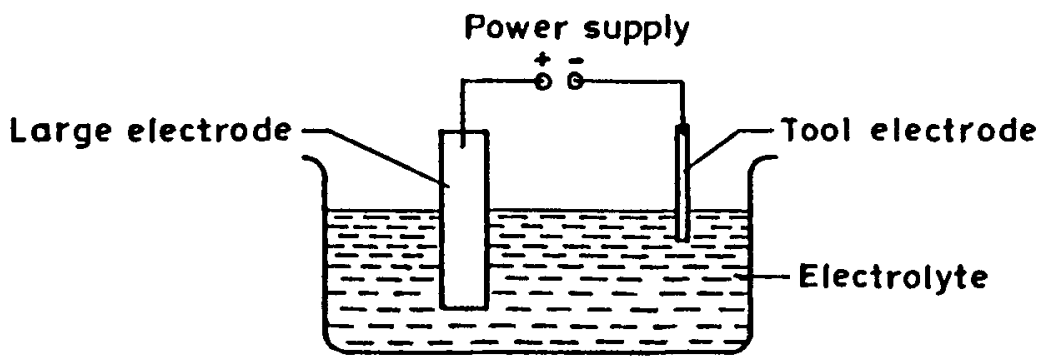

Figure 4. Schematic diagram of ECD set-up.

One phenomenon used to produce intense localized heat generation without using any sophisticated technology like electron beam or laser beam is the electrochemical discharge. Figure 4 shows an electrochemical cell where one electrode is very small and the other is relatively much larger. When these electrodes are connected to a voltage source (either AC or DC) an electrical discharge can be seen at the tip of the smaller electrode if the supply voltage exceeds a critical value. This is known as electrochemical discharge which takes place between the tip of the smaller electrode and the electrolyte in its immediate neighbourhood.

The temperature at the discharge zone has been measured by thermocouple technique (Basak 1991) and the result is shown in figure 5. This is, of course, the temperature of the thermocouple junction which itself happens to be the tip of the smaller electrode. The temperature of the discharge itself has been estimated by emission spectroscopy (Reghuram 1994) for different electrolytes with varying concentration. The temperature is found to be in the range $8000-10000 \mathrm{~K}$.

Thus, it is possible to cause material removal in very small quantities by melting and evacuation if the workpiece is kept very near the tool tip and within the range of the discharge. The work material need not be electrically conducting in this case. Such a scheme is presently in the process of development and this process is termed "Electrochemical Discharge Machining (ECDM)."

The scheme of the basic ECDM process is indicated in figure 6. The tip of the tool electrode is very close to the work surface (electrically nonconducting) and small holes can be drilled by this process. Slots can be also cut by using a knife edge tool electrode. Slots or grooves of any shape can be machined by controlling the lateral motion of the tool tip of a pointed tool.

\section{Mechanism of ECD phenomenon and ECD machining}

This process, though it appears to have considerable potential for machining electrically non-conducting materials, has very limited acceptance mainly because of its limited capacity. To improve the process capability of ECDM it is essential to understand the basic mechanism of ECDM and identify the process parameters correctly.

The process is extremely complex involving electrochemistry, heat transfer and boiling, melting and evacuation. To understand ECDM, first the phenomenon of ECD should be investigated. For this purpose an extensive study has been carried out. One of the important 


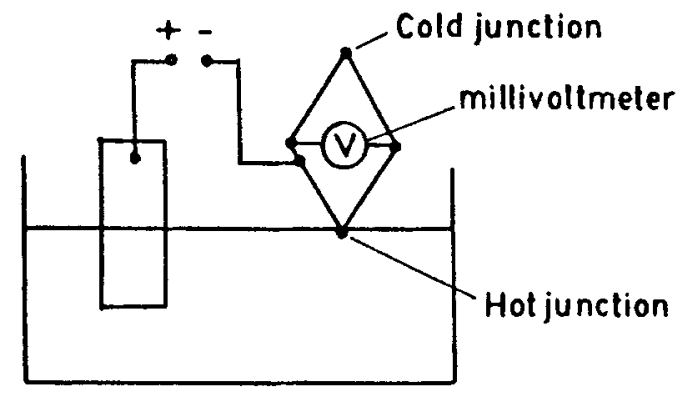

(a)

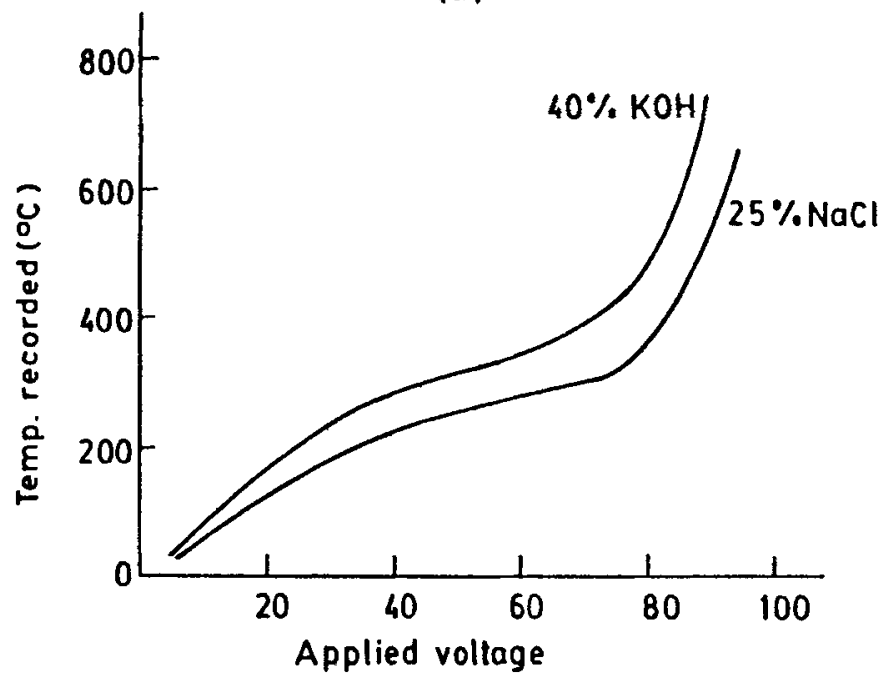

(b)

Figure 5. (a) Set-up to determine electrode temperature. (b) Result obtained.

findings is the nature of the potential drop across the electrolytic cell. A typical variation is indicted in figure 7 (Allesu 1988). It is clear that an overwhelmingly large fraction of the total drop takes place across a very thin layer surrounding the tool electrode (the smaller one) during electrochemical discharge. This suggests that a resistant layer develops around the tool electrode. In most experiments this is the cathode and, therefore, $\mathrm{H}_{2}$ evolves in the form of very fine bubbles. Moreover, the temperature of the electrolyte at this region increases because of much higher current density. This leads to boiling if the current density is high enough. Both these result in the development of a non-conducting gas and vapour blanket around the smaller electrode (this does not happen at the larger electrode because the current density is much less). When the voltage (either full-wave rectified DC or smooth DC) applied across the ECD cell is gradually increased, the corresponding change in the average current is shown in figure 8. An extensive study shows (Allesu 1988; Basak 1991; Allesu et al 1992; Reghuram 1994) that these characteristics remain unchanged for all situations. It is seen that electrochemical discharge starts only when the applied voltage reaches a critical value which depends on the type of electrolyte and its concentration. The 


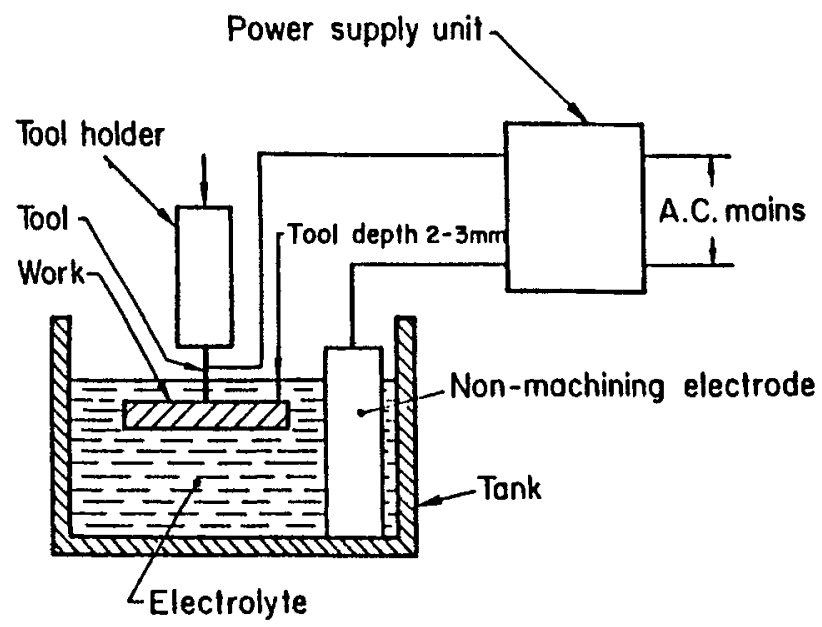

Figure 6. Configuration of non-conductor work machining using ECD.

depth to which the electrode is submerged and the electrode diameter do not appear to have much effect as long as the discharging electrode is much smaller than the other one. It is also very interesting to note that the power consumption (for a given electrolyte and set of electrodes) at the critical condition ( $\left.=V_{c r} . I_{c r}\right)$ remains constant and is independent of electrolyte concentration (Basak 1991). This strongly suggests that blanketing can be a major factor in the occurrence of electrochemical discharge as both $\mathrm{H}_{2}$ evolution and vapour production through boiling depend on the power only.

The conditions under which blanketing takes place have been investigated and a theoretical model has been developed for the ECD mechanism and for ECDM (Basak 1991; Basak \& Ghosh 1995) which can predict the critical voltage, critical average current and also the material removal rate under prescribed conditions. The results agree reasonably well with the experimental observations. This investigation also provides a new input which suggests that the electric discharge is primarily due to (Basak 1991; Basak \& Ghosh 1995) switching action and not to the breakdown of gas in the blanket as suggested by the previous researchers.

Figure 9 shows the various stages of bubble coverage of the tool surface as the applied voltage is gradually increased. At the critical condition, the hemispherical bubbles cover the surface completely. In this closed-packed condition, the in-between conducting bridges are very narrow and the current densities in these bridges become very high and cause instant boiling which blows the bridges. When a bridge is blown a spark is generated by switching action. This is why sparking takes place even if the applied voltage is much lower than the minimum voltage for breakdown as indicated in the Paschen curve. Figure 10 shows the equivalent circuits. As switching action plays the crucial role in spark generation, inductance in the circuit becomes one of the most important parameters. This however remained unnoticed so far. This finding thus leads to an extra process parameter which can be easily controlled and a substantial improvement in the process capability of ECDM can be achieved. 


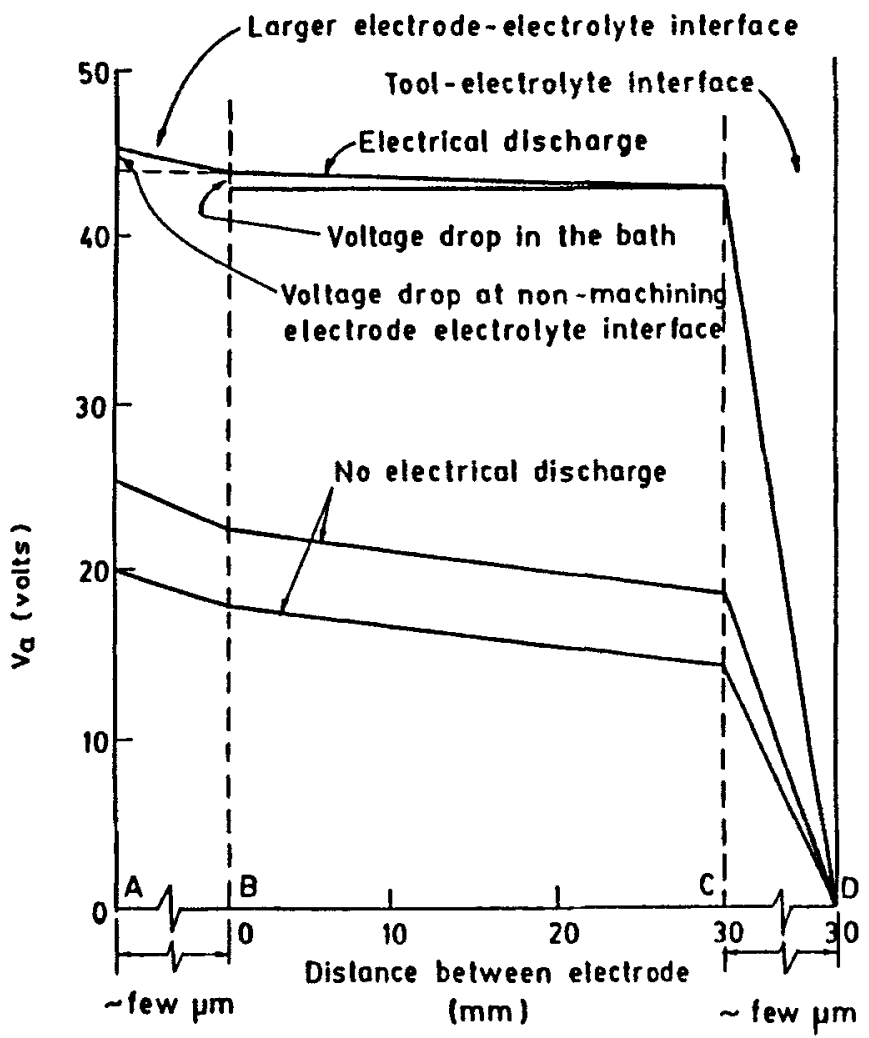

Figure 7. Distribution of voltage drop in an ECDM bath [Electrolyte : $\mathrm{NaOH}(35 \%)$; tool polarity: -ve; power supply: DC].

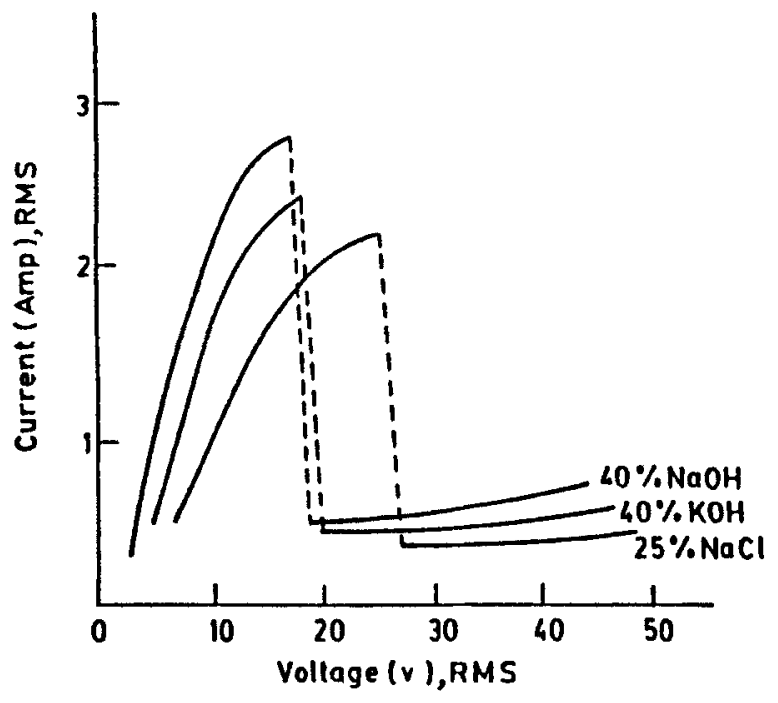

Toal dia: $0.11 \mathrm{~cm} \quad$ Tool depth: $:=0.2 \mathrm{~cm}$

Power supply: Smoolh D.C.

Figure 8. $\quad V-I$ characteristics for different electrolytes. 


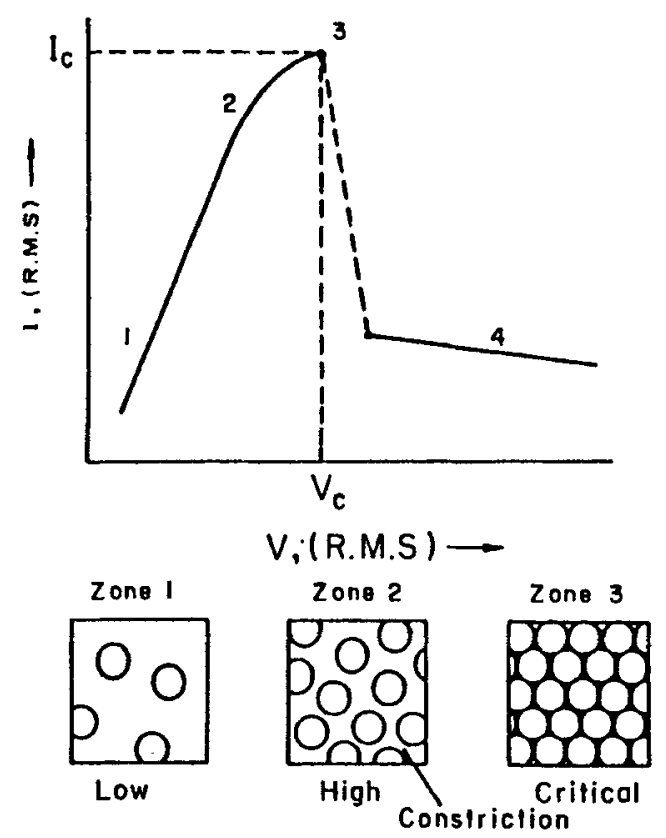

Figure 9. Bubble density on tool electrode at different applied voltages.
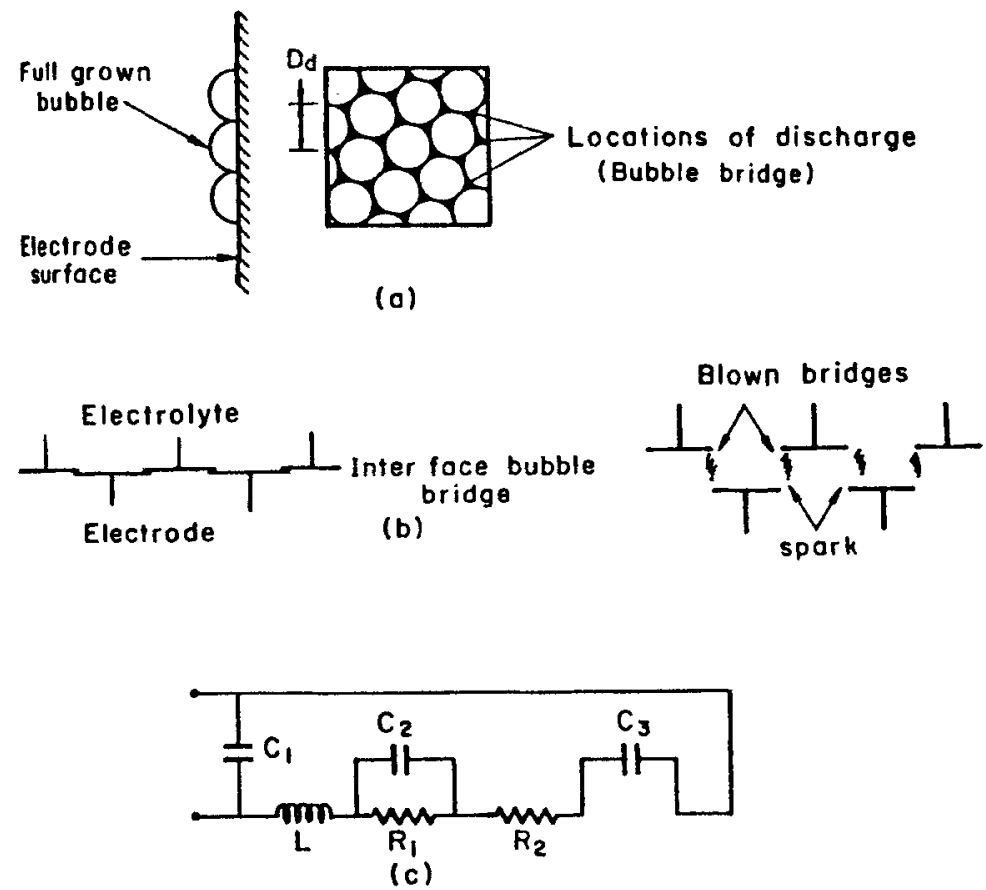

Figure 10. (a) Discharge locations with bubble distribution at critical condition. (b) Idealised switching off situation, and (c) Idealised equivalent circuit at discharge. 


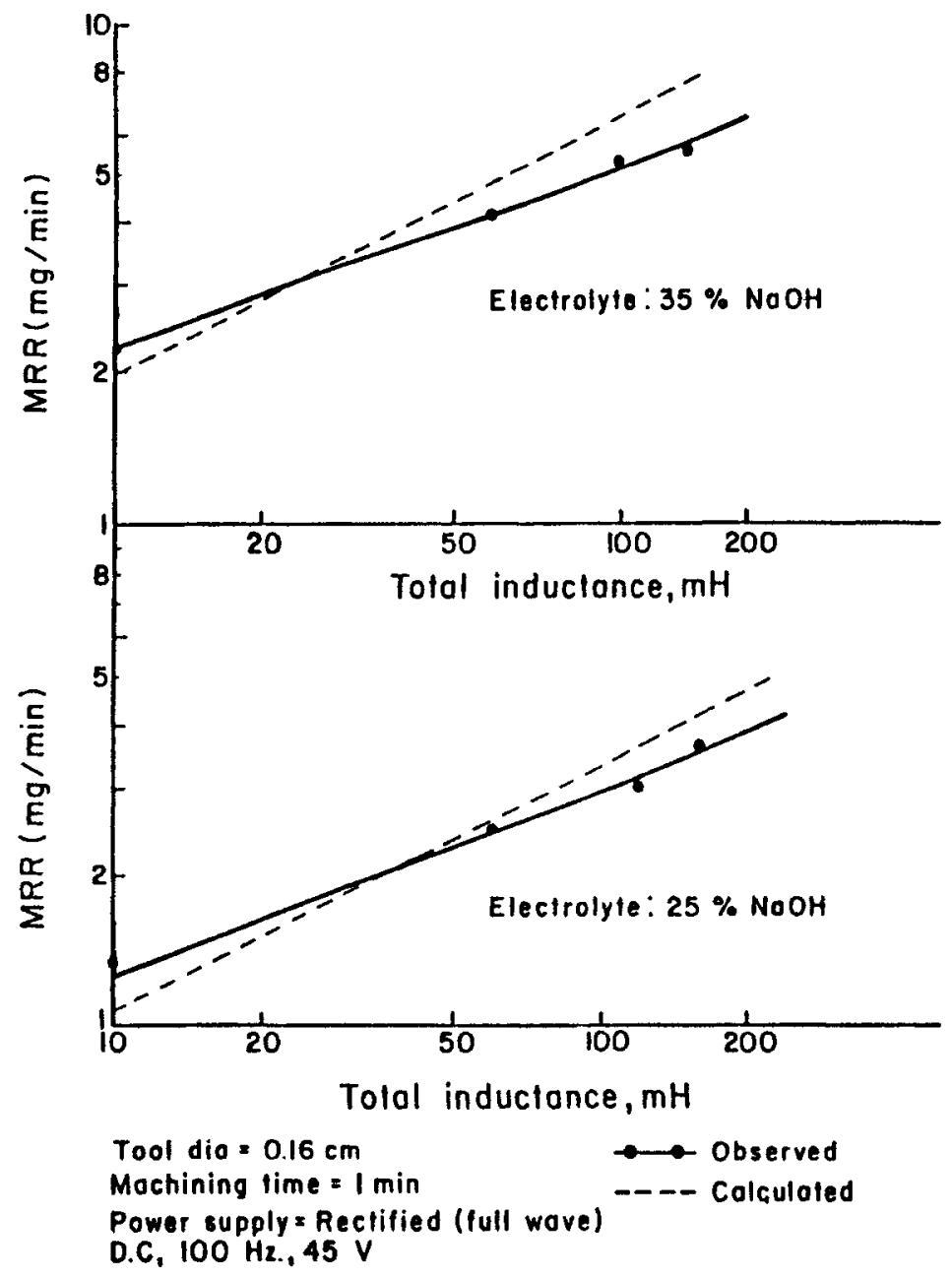

Figure 11. Total inductance vs $\mathrm{MRR}(\mathrm{NaOH})(\log$ scale, workpiece - glass).

\section{Improvement of process capability}

A number of experiments on ECDM have been conducted using artificially introduced inductance in the circuit (Basak 1991; Basak \& Ghosh 1995b). It has been observed that increasing the inductance in the circuit results in higher material removal rate. The increase is quite significant and a set of sample results is shown in figure 11. Further investigation can provide more interesting results in this direction.

The idea of using artificially introduced inductance in an ECDM circuit has been put forward (Reghuram 1994) in a somewhat different direction. It has been found that when smooth DC voltage is applied, the introduction of a series inductance of adequate value in the circuit can lead to a "total discharge condition." In this condition the discharge appears like a flame emerging from the tool tip. To obtain a stable total discharge without the undesirable disturbance in the electrolyte, the input voltage is increased to a high value $(\approx 100 \mathrm{~V})$ to obtain stable discharge. Once the stable discharge is attained the input voltage 


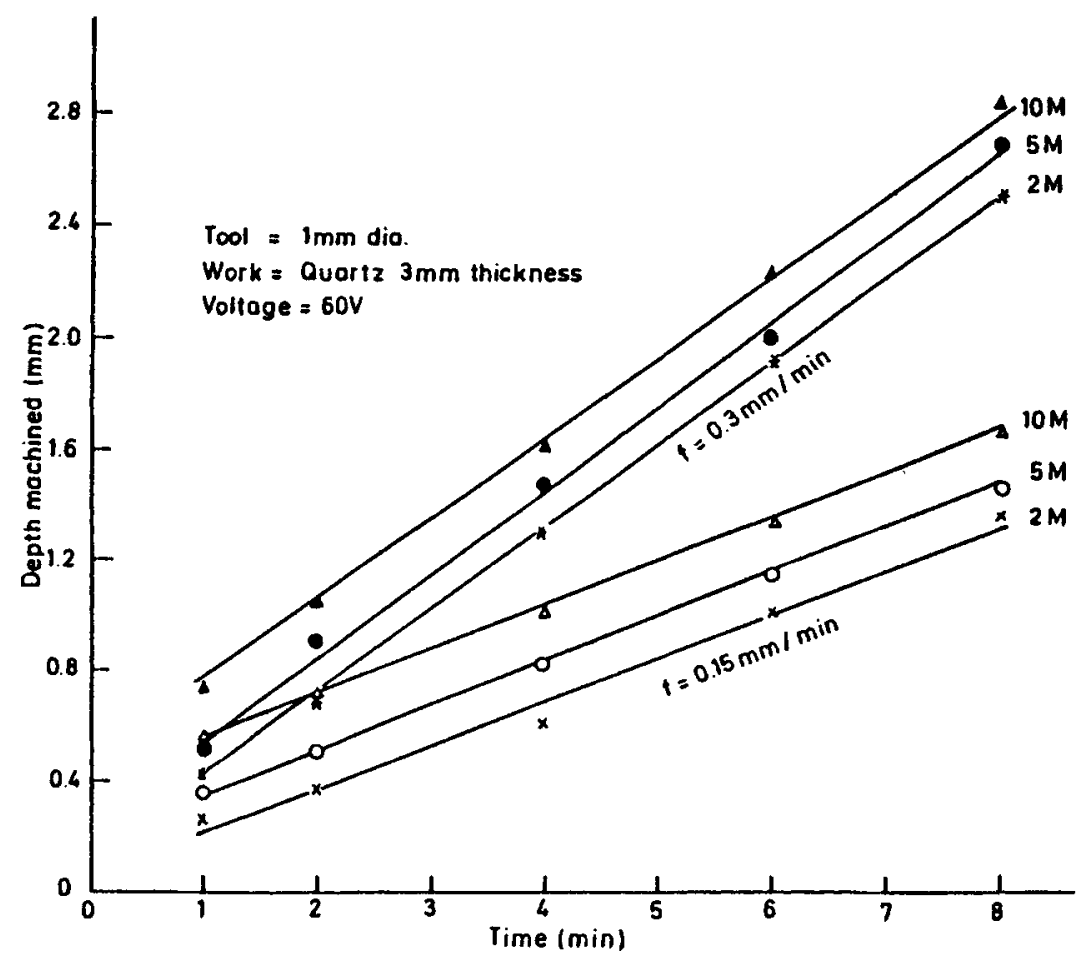

Figure 12. Variation of machined depth with time [electrolyte: $\mathrm{KOH}$ ].

is lowered to the operational value $(\approx 60 \mathrm{~V})$ and it is found that the energy input to the system is much higher.

Under this situation it is not necessary to keep the workpiece in contact with the tool tip as is done in the common ECDM. It is enough to keep the work surface within the range of the stable discharge; the process can be considered to be somewhat analogous to laser-beam machining. However, gravity feed is no longer possible in this type of ECDM and the tool has to be fed by a servo drive as is done in EDM. Figure 12 shows that by employing this modified ECDM holes can be drilled in a quartz block with a penetration rate of $0.3 \mathrm{~mm} / \mathrm{min}$ and can be continued even if the tip reaches depth of the order of $3 \mathrm{~mm}$ or more. This has not been possible with the usual ECDM without a series inductance.

\section{Other manufacturing applications}

At this point it is worth mentioning a few other applications of ECD in manufacturing processes. Using this phenomenon a scheme for scribing.on non-conducting materials has been developed (Allesu 1988). A scribing pen to write on glass and ceramics was fabricated and it performed satisfactorily.

Another important manufacturing application of ECD phenomenon besides machining is microwelding. It has been shown (Allesu 1988) that micro-welding of a fine thermocouple can be very conveniently performed using the heat generated by ECD. The scheme is 


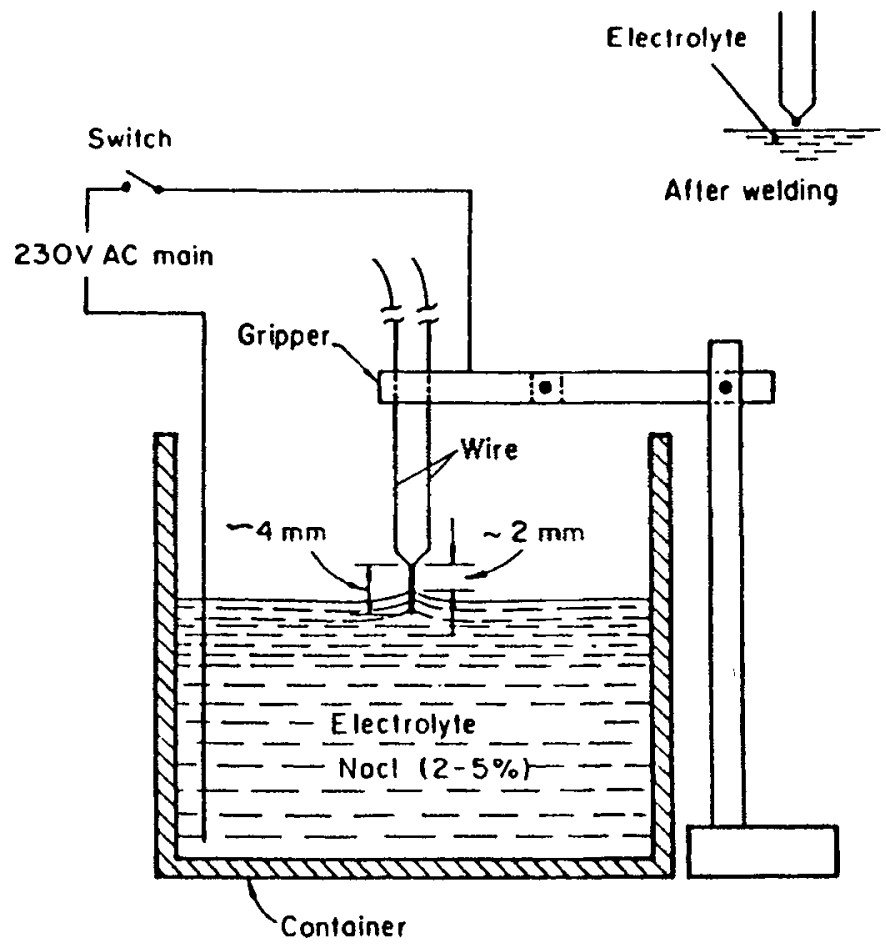

Figure 13. Schematics of micro-welding set-up.

very simple and is shown in figure 13. The process has been investigated in detail (Parija 1993; Ghosh et al 1995) and it was possible to determine the optimal combinations of the process parameters for obtaining excellent welds. Figure 14 shows the suitable regions of the voltage-current combination which yield good results. The work is still in progress.

Since a controlled localized heating is possible with the help of electrochemical discharge other important processing methods can be developed. Surface treatment using the pointed heat source is one such example. A more interesting application of the ECD phenomenon can be "Rapid Prototyping" with fused deposition of very thin metallic wires as indicated schematically in figure 15 . Currently rapid prototyping using metals instead of polymers, resins, plastics etc. are done using high power lasers and the system is expensive. A low budget metallic fused deposition modelling type rapid prototyping may be possible using ECD.

\section{Concluding remarks}

The present investigations indicate that ECD can be a very useful tool for different types of manufacturing processes. The mechanism of spark generation is found to be primarily the switching phenomenon rather than the breakdown of insultaing gas layer. This understanding resulted in a major improvement of the process capability by artificially introducing a suitable amount of inductance in the circuit. Further research resulted in a new scheme of ECDM with much higher penetration capability. Besides machining, the 


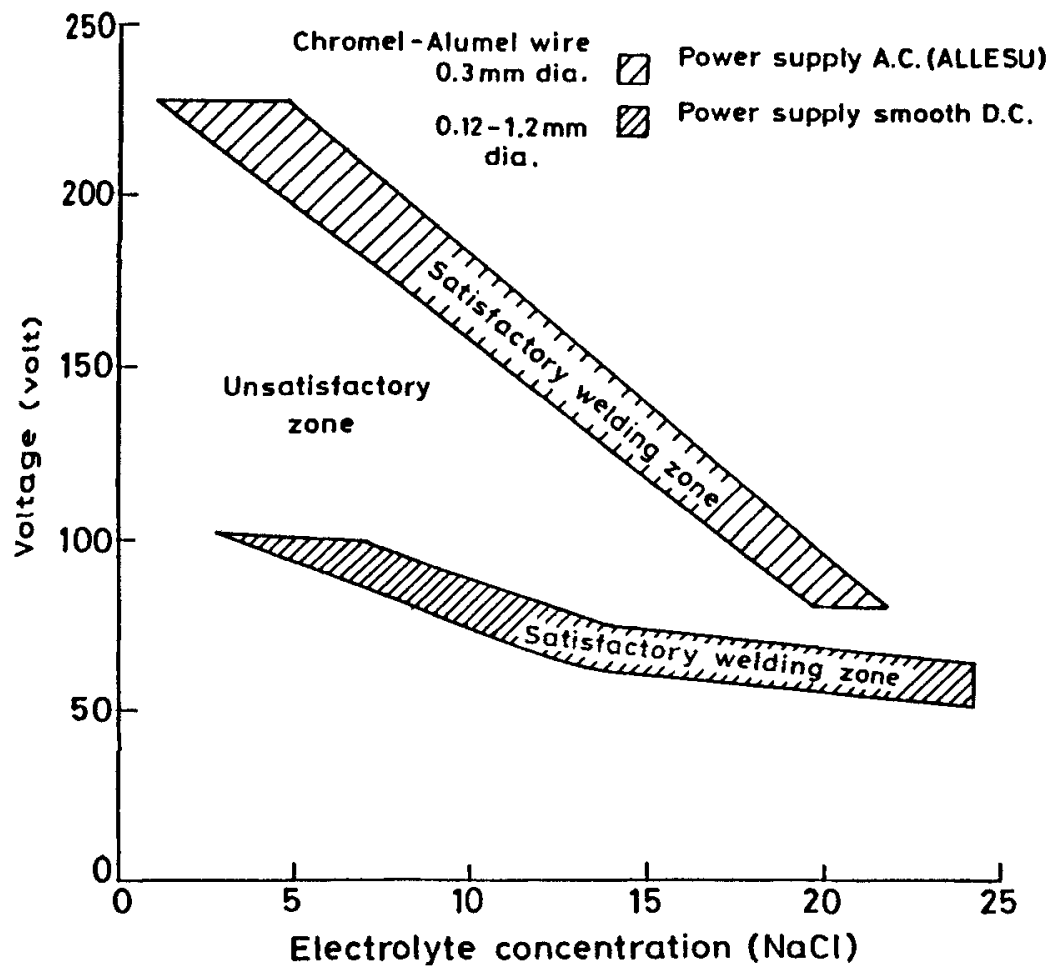

Figure 14. Nature of voltage - electrolyte concentration in microwelding.

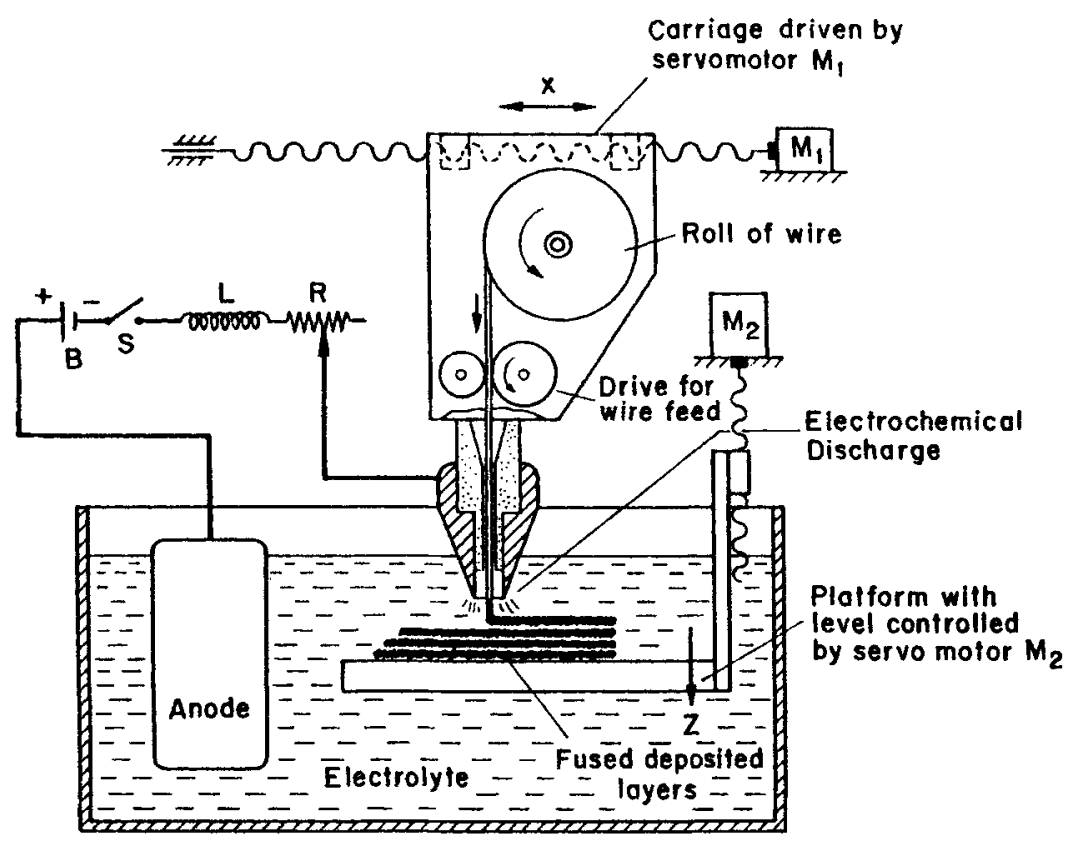

Servodrive for the $y$ - direction not shown

Figure 15. Scheme of ECD fused deposition modelling. 
ECD phenomenon has been very effectively used for micro-welding. ECD micro-welding is a very simple process and can be useful to the industry. Similarly there is a great potential for ECD in other types of material processes and fused deposition modelling for rapid prototyping.

\section{References}

Allesu K 1988 Electrochemical discharge phenomenon in manufacturing processes. $\mathrm{Ph} \mathrm{D}$ thesis, Indian Institute of Technology, Kanpur

Allesu K, Ghosh A, Muju M K 1992 A preliminary quantitative approach of a proposed mechanism of material removal in electrical machining of glass. Eur. J. Mech. Eng. 36: 202-207

Basak I 1991 Electrochemical discharge machining mechanism and a scheme for enhancing material removal capacity. $\mathrm{Ph} \mathrm{D}$ thesis, Indian Institute of Technology, Kanpur

Basak I, Ghosh A 1995a Mechanism of spark generation during electrochemical discharge machining. J. Mater. Process. Technol. 62: 46-53

Basak I, Ghosh A 1995b Mechanism of material removal in electrochemical discharge machining: A theoretical model and experimental verification J. Mater. Process. Technol. (in press)

Ghosh A, Muju M K, Parija S, Allesu K 1995 Microwelding using electrochemical discharge. Int. J. Machine Tools Manuf. (in press)

Parija S 1993 A thermal model and parametric investigation of electrochemical discharge microwelding. M Tech thesis, Indian Institute of Technology, Kanpur

Reghuram V 1994 Electrical and spectroscopic investigations in electrochemical discharge machining. Ph D thesis, Indian Institute of Technology, Madras and Indian Institute of Technology, Kanpur 